\title{
Flexible nine-channel photodetector probe facilitated intraspinal multisite transcutaneous photobiomodulation therapy dosimetry in cadaver dogs
}

Daqing Piao

Lara A. Sypniewski

Christian Bailey

Danielle Dugat

Daniel J. Burba

Luis De Taboada 


\section{Flexible nine-channel photodetector probe facilitated intraspinal multisite transcutaneous photobiomodulation therapy dosimetry in cadaver dogs}

\author{
Daqing Piao, ${ }^{a, b, \star}$ Lara A. Sypniewski, ${ }^{b}$ \\ Christian Bailey, ${ }^{a}$ Danielle Dugat, ${ }^{\text {b }}$ \\ Daniel J. Burba, ${ }^{b}$ and Luis De Taboadac \\ ${ }^{a}$ Oklahoma State University, School of Electrical and \\ Computer Engineering, Stillwater, Oklahoma, United States \\ bOklahoma State University, Department of Veterinary Clinical \\ Sciences, Center for Veterinary Health Sciences, Stillwater, \\ Oklahoma, United States \\ 'LiteCure LLC, Carlsbad, California, United States
}

\begin{abstract}
Noninvasive photobiomodulation therapy (PBMT) of spinal cord disease remains speculative due to the lack of evidence for whether photobiomodulatory irradiances can be transcutaneously delivered to the spinal cord under a clinically acceptable PBMT surface irradiation protocol. We developed a flexible nine-channel photodetection probe for deployment within the spinal canal of a cadaver dog after hemilaminectomy to measure transcutaneously transmitted PBMT irradiance at nine sites over an eight-cm spinal canal length. The probe was built upon a $6.325-\mathrm{mm}$ tubular stem, to the surface of which nine photodiodes were epoxied at approximately $1 \mathrm{~cm}$ apart. The photodiode has a form factor of $4.80 \mathrm{~mm} \times 2.10 \mathrm{~mm} \times$ $1.15 \mathrm{~mm}$ (length $\times$ width $\times$ height). Each photodiode was individually calibrated to deliver $1 \mathrm{~V}$ per $7.58 \mu \mathrm{W} / \mathrm{cm}^{2}$ continuous irradiance at $850 \mathrm{~nm}$. The outputs of eight photodiodes were logged concurrently using a data acquisition module interfacing eight channels of differential analog signals, while the output of the ninth photodiode was measured by a precision multimeter. This flexible probe rendered simultaneous intraspinal (nine-site) measurements of transcutaneous PBMT irradiations at $980 \mathrm{~nm}$ in a pilot cadaver dog model. At a surface continuous irradiance of $3.14 \mathrm{~W} / \mathrm{cm}^{2}$ applied off-contact between L1 and L2, intraspinal irradiances picked up by nine photodiodes had a maximum of $327.48 \mu \mathrm{W} / \mathrm{cm}^{2}$ without the skin and $5.68 \mu \mathrm{W} / \mathrm{cm}^{2}$ with the skin. $\odot$ The Authors. Published by SPIE under a Creative Commons Attribution 3.0 Unported License. Distribution or reproduction of this work in whole or in part requires full attribution of the original publication, including its DOI. [DOI: 10.1117/1.JBO.23.1.010503]
\end{abstract}

Keywords: photobiomodulation therapy; dosimetry; spinal cord injury; low-level light therapy.

Paper 170710LR received Nov. 2, 2017; accepted for publication Dec. 28, 2017; published online Jan. 23, 2018.

*Address all correspondence to: Daqing Piao, E-mail: daqing.piao@okstate .edu

\section{Introduction}

Photobiomodulation therapy (PBMT), ${ }^{1}$ previously termed lowlevel light therapy, ${ }^{2}$ has shown safe and potentially beneficial therapeutic outcomes for conditions including stroke ${ }^{3}$ and wounds that resist conventional treatments. ${ }^{4}$ The regenerative responses of the axon under photobiomodulatory doses of light, ${ }^{5}$ most commonly at $810 \mathrm{~nm}$ in studies performed on rodent models, ${ }^{6-8}$ are particularly promising for treating spinal cord diseases that are difficult to access anatomically and therapeutically. However, in spite of the obvious potential for the treatment regimen, PBMT for spinal cord disease remains clinically untested. The lack of enthusiasm of PBMT for spinal cord conditions roots in the absence of experimental dosimetric data ${ }^{9}$ suggesting that photobiomodulatory irradiances could be successfully delivered to the spinal cord level via transcutaneous transmission under a surface irradiation protocol that will not cause collateral thermal damage.

Accurate radiometry is central to energy-based therapeutic applications. $^{10,11}$ To our knowledge, no study has reported the skin-to-spinal-cord light transmission of a photobiomodulatory dose that is translatable to humans. Previously, a single photodiode sensor was used to sequentially measure transcutaneous light transmission of 980-nm irradiation to five vertebral locations including T12-13, T13-L1, L1-2, L2-3, and L3-4 in a cadaver dog. ${ }^{12}$ The $0.00102 \%$ transcutaneous light transmission to the shallowest level of the spinal canal of the dog was comparable to the $0.00114 \%$ transcranial light transmission measured from the same subject. The transcranial light transmission of $0.00114 \%$ in this dog cadaver also agreed with transcranial light transmission at 5-cm depth in human cadavers. ${ }^{1}$ It is, thus, perhaps possible to deliver PBMT light to the spinal cord level at a dose that has shown the potential of improving human cerebral conditions ${ }^{13}$ at a clinically safe surface irradiation protocol. However, the use of a single photodiode sensor challenges the localization of the probe once deployed intraspinally, the consistency of multisite measurements on any subject, and the reproducibility of inter-subject evaluation that are all parametric to the feasibility of noninvasive PBMT of the spinal cord under a clinically acceptable surface light irradiation protocol. In this letter, we report the development of a flexible ninechannel probe, $\sim 80$-mm long, for deployment in the spinal canal of large cadaver dogs after hemilaminectomy and demonstrate simultaneous intraspinal light irradiance quantification at nine sites under surface light irradiation. The probe had nine photodiodes (PDs) for absolute light irradiance measurements. The probe also had nine fiber-optical diffusers (FODs) as auxiliary sensors for relative scaling of the light irradiations reaching the nine PDs at low light irradiance conditions.

\section{Probe and Interfacing Instrument}

Figure 1 shows the sizes of the PD and FOD sensors (A), the sensor configuration on the probe (B), the key fabrication procedures of the probe $(\mathrm{C})$, and the completed module of the probe (D) with the PDs and FODs interfaced to custom readout instruments. The PDs were silicon PIN photodiodes (SFH2400FA-Z, OSRAM Opto Semiconductors GmbH, Regensburg, Germany) with a spectral response from 750 to $1100 \mathrm{~nm}^{12}$ covering the common PBMT wavelengths. The PD had a form factor of $4.80 \mathrm{~mm} \times 2.10 \mathrm{~mm} \times 1.15 \mathrm{~mm}$ (length $\times$ width $\times$ height). The FODs were cylindrical diffusers with a form factor of $1.75 \mathrm{~mm} \times 0.20 \mathrm{~mm}$ (length $\times$ diameter). One PD and one FOD were placed lateral to each other to form one of the nine sets of 


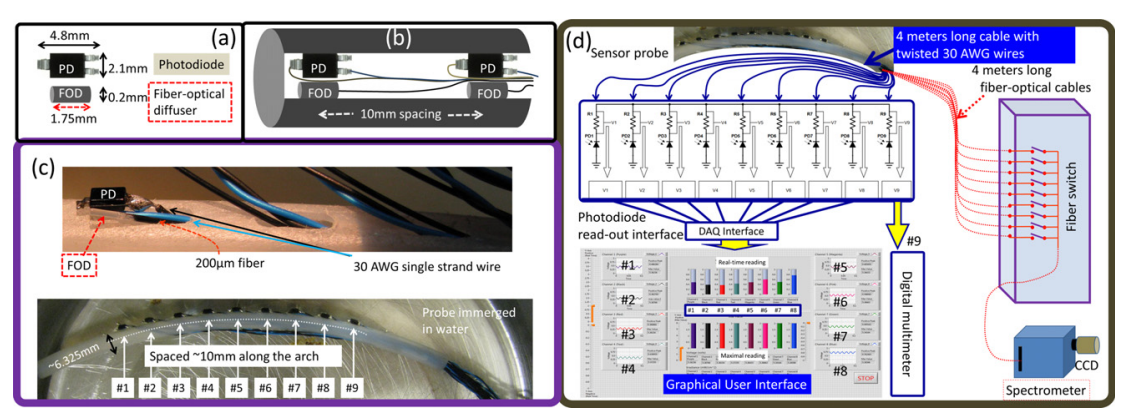

Fig. 1 (a) The photodiode (PD) and the auxiliary cylindrical fiber-optical diffusor (FOD). (b) One PD and one FOD formed a set of photodetecting sensors placed $10 \mathrm{~mm}$ apart from its nearest neighbors. (c) Each PD was cabled with a pair of 4-m-long 30 AWG single-strand wires and each FOD was coupled to a 4-m-long fiber of $200-\mu \mathrm{m}$ core. Each set of the paired wires and the fiber were passed through an aperture on the plastic tubular substrate for connecting to the readout equipment. (d) The nine PDs were wired to a nine-channel conditioning circuit module, of which eight channels were wired to an eight-channel differential input data acquisition module controlled by a GUI (a screenshot of the GUI is shown), and the ninth channel was wired to a precision digital multimeter. The nine auxiliary FOD sensors were interfaced to a spectrophotometer via a $9 \times 1$ fiber switch.

photodetector sensor combinations on the flexible probe. Each PD was terminated with a twisted pair of 4-m-long 30 AWG single-strand wires. Each FOD was coupled to a 4-m-long $200-\mu \mathrm{m}$ optical fiber (Pioneer Optics, Bloomfield, Connecticut) terminated with an SMA-905 connector. The flexible probe was fabricated using a 150-mm-long plastic tubular substrate with an outer diameter of $6.325 \mathrm{~mm}$. Nine identical through-apertures (5-mm long, 2-mm wide, and $10 \mathrm{~mm}$ apart) were made along the substrate wall for aligning the photodetector sensors and passing the cables. Ultraviolet curing optical epoxy (NOA61, Edmond Optics, Barrington, New Jersey) was used to completely cover the PD including the terminals and the FOD. The electrical insulation among the nine PDs was examined by measuring the intra-channel conductance of any single PD and interchannel leakage between any two PDs when the probe was immersed in water.

The nine pairs of the 4-m-long electrical wires cabling the nine PDs and the nine sets of 4-m-long optical fibers coupling the nine FODs were braided together and connected to the PD interfacing module and the FOD readout system. The PD's 4-m-long wires were connected to a circuit interfacing box through a 25-pin D-connector. A stand-alone custom linear regulated power supply provided the required $5 \mathrm{~V}$ reversed bias voltage for all nine PDs. The photocurrent of each PD was converted to a voltage signal by a scaling resistor. The scaling resistors were inserted into sockets on a circuit board instead of soldered to the circuit board to allow for easy resistor replacement during calibration and tissue measurements. Signals from eight of the nine PD readout channels were connected, via a second 25-pin D-connector on the box, to an NI (National Instruments, Austin, Texas) SCB-68A connector block and cabled to an NI PCIe-6321 data acquisition (DAQ) board housed in a desktop computer (Dell Optiplex 7020, Round Rock, Texas). The DAQ board had a capacity to acquire eight channels of differential analog inputs. The eight-channel PD signals interfaced to the DAQ board were monitored concurrently and in real time on a graphical user interface (GUI) developed in the LabVIEW platform (National Instruments, Austin, Texas). The GUI registered the peak output of each PD channel in addition to displaying the instantaneous output. The logging of the instantaneous and peak readings from the PDs was important when light transmission measurements in tissue were made at high surface light irradiance, which could only be briefly applied (a couple of seconds at the $10 \mathrm{~W}$ continuous irradiances used in this study on cadaver dog tissue) to prevent collateral thermal tissue damage that could change the optical tissue characteristics for follow-up readings. The ninth PD readout channel was connected via a BNC cable to a precision multimeter (Hewlett Packard 3478A, Palo Alto, California) for manual recording. The 4-m-long 200- $\mu \mathrm{m}$ optical fibers of the nine FOD sensors were coupled to nine $200-\mu \mathrm{m}$ input channels on a $9 \times 1$ fiber switch (Piezosystem, Jena, Germany) for routing sequentially to one $200-\mu \mathrm{m}$ output channel, which was coupled via a custom optical fiber adaptor to a spectrometer of 300-mm focal length (SpectroPro2300i, Acton Research, Trenton, New Jersey) for data acquisition by an intensified charge-coupled-detector (CCD) camera (PI-Max, Princeton Instruments, Trenton, New Jersey). The CCD camera was controlled by a second desktop computer (Dell Optiplex GX520, Round Rock, Texas) using a WinSpect interface. The CCD acquisition, which was controlled with various settings of gain and exposure time, rendered a much greater dynamic range than the PD readout circuit could produce.

\section{Probe Calibration}

Figure 2 shows the linearity performances of the nine PDs on the probe when examined in free-space using the test-bench setup shown in (a) and (b), schematic and photograph, respectively. The test-bench was designed to allow for the irradiance responses of each PD on the probe to be compared repeatedly and rapidly against a commercial power-meter system (PM200, Thorlabs Inc., Newton, New Jersey) containing a silicon sensor (S130c) for light irradiations varying by more than four orders of magnitude. The continuous output of an 850-nm diode laser controlled by a temperature control module (Throlabs TED 200C, Newton, New Jersey) and a laser-diode driver (Thorlabs LDC 205C, Newton, New Jersey) was fiber-coupled to a beam projection module (BPM) on the test bench. The BPM collimated the fiber-output using a lens system and homogenized the collimated beam's irradiance distribution using a $10 \mathrm{deg}$ holographic diffuser window (Edmund Optics, Barrington, New Jersey), before projecting the homogenized beam with a tunnel-aperture of $1.6 \mathrm{~mm}$ in diameter and $115 \mathrm{~mm}$ in length. The homogenized beam exiting the tunnel-aperture was directed to one of the nine PDs on the probe, which was mounted on a stage-system providing $5 \mathrm{deg}$ of motion (three translational and two rotational). The lateral, elevational, and longitudinal positions of each PD and two angular orientations of the light receiving facet of the $\mathrm{PD}$ were all adjusted to optimize the light 


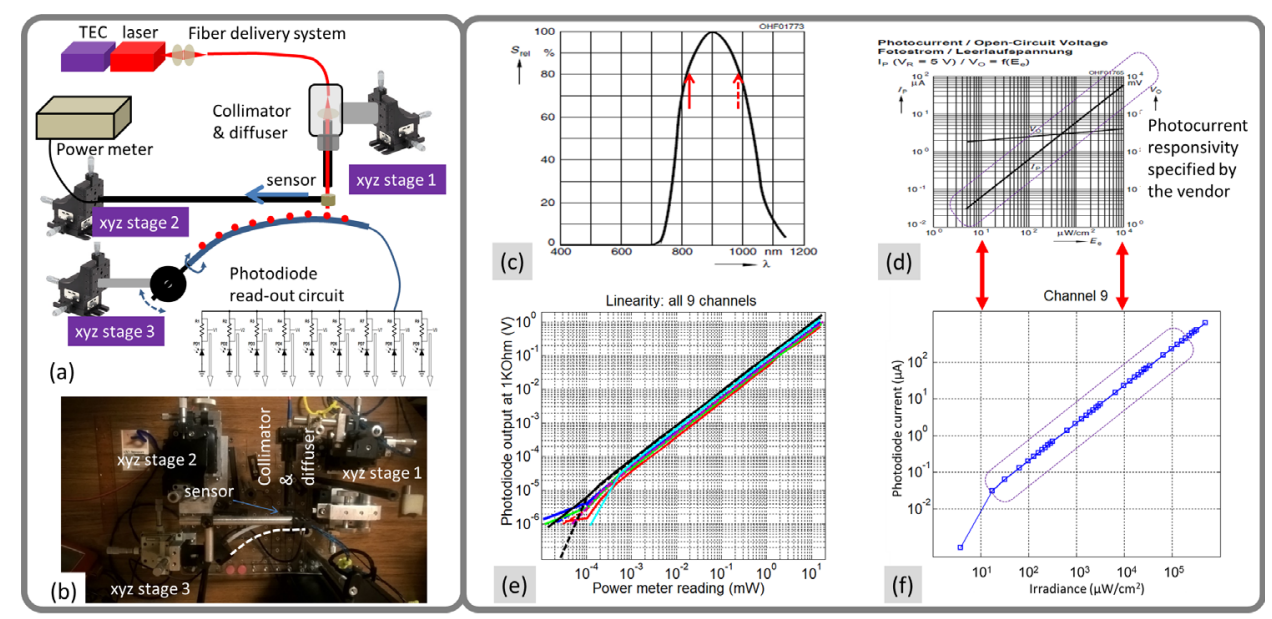

Fig. 2 (a) The configuration of the free-space test-bench for quality-checking of the nine PDs on the applicator probe. The BPM had four degrees of freedom. The beam output of BPM was collimated and then homogenized by a diffuser. The homogenized beam was further collimated and aligned to the PD light receiving surface. A commercial silicon sensor connected to a power meter was positioned to intercept the collimated beam before reaching the PD. (b) The top-view photograph of the free-space test bench. (c) The spectral response of the PD. The solid arrow marks the wavelength $(850 \mathrm{~nm})$ of the laser delivered to the test bench. The dashed arrow marks the wavelength $(980 \mathrm{~nm})$ of the PBMT laser used for studies on the dog. (d) The vendor-provided irradiance response of the PD at $5 \mathrm{~V}$ bias voltage. (e) The responses of all nine PDs over four orders of magnitude of the irradiating power. (f) The irradiance response of the PD \#9 plotted with the same scales as that of (d). The two arrows are for aligning the two plots. Linear responses from all nine PDs were achieved over four decades of the irradiances.

receiving angle of the PD. The commercial silicon sensor of the PM200 system with an active window of $1 \mathrm{~cm}$ in diameter was positioned to intercept the illuminating beam in front of the PD being tested. The silicon sensor was mounted on a stagestructure that allowed it to be quickly removed from the illuminating beam for taking the reading from the illuminated PD on the probe, and then quickly returned to its original position (back to the line of sight of the beam path) for acquiring the reference reading of the same beam from the commercial power meter. The vendor-specified spectral and photocurrent responses of the PD (at $870 \mathrm{~nm}$ ) are given in (c) and (d), respectively. The responses of each of the nine PDs were measured by adjusting the continuous power of the homogenized 850-nm illumination beam at the levels including at least the following values: $0.1 \mu \mathrm{W}$, $0.2 \mu \mathrm{W}, 0.5 \mu \mathrm{W}, 1 \mu \mathrm{W}, 2 \mu \mathrm{W}, 5 \mu \mathrm{W}, 10 \mu \mathrm{W}, 20 \mu \mathrm{W}, 50 \mu \mathrm{W}$, $100 \mu \mathrm{W}, 200 \mu \mathrm{W}, 500 \mu \mathrm{W}, 1 \mathrm{~mW}, 2 \mathrm{~mW}, 5 \mathrm{~mW}$, and $10 \mathrm{~mW}$. The responses of all nine PDs, using a $1 \mathrm{~K} \Omega$ scaling resistor for the current-to-voltage conversion, over more than four decades of the continuous light irradiation power are given in (e). All nine PDs had a linearity of better than 0.9998 over the entire range of light irradiation measurements. An example of the calculated photocurrent of PD \#9 versus the referenced light irradiation was plotted in (f), using the scales the same as those shown in the vendor-provided photocurrent specifications of (d).

The probe was also placed in a tank containing $~ 51$ of $1 \%$ intralipid solution diluted from a $20 \%$ bulk solution (Baxter Healthcare, Deerfield, Illinois), and a spherical light diffuser SD200 (Medlight SA, Switzerland) fixed to a 100-mm-long 4-mm diameter stainless-steel tubing was positioned lateral to the native plane of the probe substrate and aligned approximately to PD \#5, for isotropic illumination at $850 \mathrm{~nm}$ using the same laser source used for the linearity tests of Fig. 2. The light irradiance measured by the nine PDs agreed very well with theoretical predictions according to photon diffusion in an infinite medium geometry and considering the positions of the nine PDs with respect to the single isotropic source. The responses of the nine-channel probe were also measured after embedding in a preserved porcine lung tissue (BioQuest, Fort Atkinson,
Wisconsin) that was pressed by a 5-cm-thick solid tissue phantom (two pieces of F0399, INO, Quebec, Canada). Contact illumination was delivered by a PBMT conical treatment head held against the solid phantom by an articulated arm and connected to a companion laser unit (LiteCure, Carlsbad, California) delivering a continuous irradiation of 0 to $15 \mathrm{~W}$ with a minimal $0.5 \mathrm{~W}$ increment at $980 \mathrm{~nm}$. According to the vendor-provided specifications, the PD's spectral response at $980 \mathrm{~nm}$ for PBMT was nearly identical to that at $850 \mathrm{~nm}$ used for the linearity calibration. The light irradiances were measured by the nine PDs within the lung-tissue under $3.14 \mathrm{~W} / \mathrm{cm}^{2}$ continuous surface irradiance (corresponding to the laser unit setting at $10 \mathrm{~W}$ power). The light irradiances measured by the $\mathrm{PD} \# 9$ in the lung tissue demonstrated the linear response of the probe to continuous surface powers of 0 to $10 \mathrm{~W}$ at a minimal of $0.5 \mathrm{~W}$ steps.

\section{Pilot Results of Multisite Dosimetry of Transcutaneous Light Transmission}

Figure 3 shows the intraspinal irradiances of transcutaneous transmission of 980-nm PBMT light at the nine PD sites over an $8-\mathrm{cm}$ span of the spinal canal of a cadaver dog (the protocol was exempted from institutional review). One cadaver (Rottweiler mix) dog of $\sim 20 \mathrm{~kg}$ weight was acquired for this study from a regional animal shelter after it was euthanized due to a terminal condition not affecting the dermis, musculature, bones, or nervous system as part of the normal animal control protocol for that shelter. With a left hemilaminectomy, the spinal canal extending from $\sim \mathrm{T} 10$ to L6 vertebral sections was exposed. The flexible probe was embedded within the exposed spinal canal (A), with the epoxying cover of the PDs placed against the dorsal wall of the spinal canal lumen for the PD's optical axis to face toward the light illumination direction (the inset showing the geometry of the cross section). The probe was constrained by its own tension inside the spinal canal, assuring a good contact between the nine PDs (bulged above the surface of the plastic tubular substrate) and the inner vertebral surface. After deploying the probe within the spinal canal, peripheral muscle and fascia tissues were pulled over and closed directly 

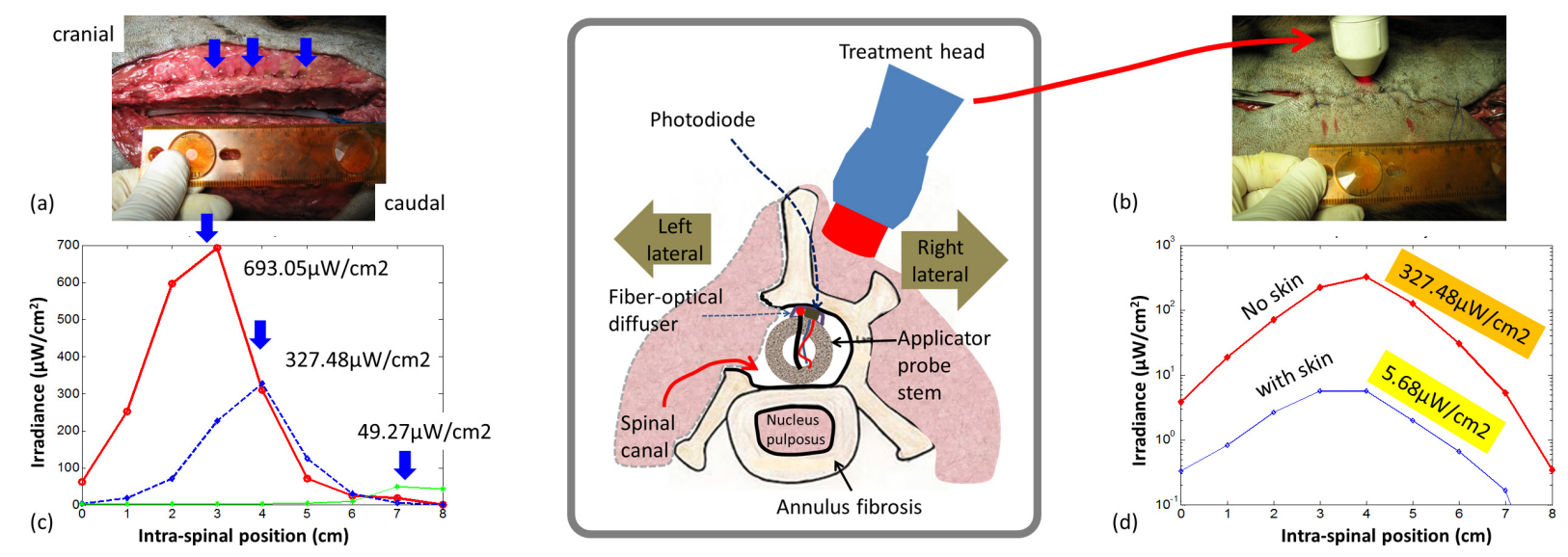

Fig. 3 (a) The applicator probe was deployed intraspinally for measuring transcutaneously transmitted 980-nm light. The hypodermic needles were used to mark the approximate locations of the nine PDs. (b) The PBMT laser conical treatment head was placed $1 \mathrm{~cm}$ from the skin above the tissue area containing intraspinally deployed probe. (c) The irradiances measured by the nine PDs in the spinal canal when surface irradiance of $3.18 \mathrm{~W} / \mathrm{cm}^{2}$ was applied without the skin to the positions aligning with PD \#4, PD \#5, and PD \#8. (d) The irradiances measured by the nine PDs in the spinal canal when surface irradiance of $3.18 \mathrm{~W} / \mathrm{cm}^{2}$ was applied without (top trace) or with (bottom trace) the skin to the positions aligning with PD \#5.

over the probe to provide a tissue environment for the photo detectors. Surface light illumination was applied by positioning the PBMT's laser conic treatment head at 1-cm distance from the tissue over a position dorsal-right-lateral to the fourth, fifth, or eighth PD (C) with the PD positions marked by hypodermic needles placed along the vertebra. All measurements performed on the dog cadaver used $22 \mathrm{M} \Omega$ current-to-voltage photodiode scaling resistors to provide a photodiode light irradiance response of $1 \mathrm{~V}$ per $7.58 \mu \mathrm{W} / \mathrm{cm}^{2}$ at $980 \mathrm{~nm}$. At a surface continuous light irradiance of $3.14 \mathrm{~W} / \mathrm{cm}^{2}$ applied at a position dorsal-lateral to PD \#4 (between T13 and L1), the intraspinal light irradiances at the nine sites peaked at PD \#4 at $0.693 \mathrm{~mW} / \mathrm{cm}^{2}$, corresponding to a light transmission of $0.02 \%\left(2.2 \times 10^{-4}\right)$. The intraspinal light irradiances at the nine sites in the absence and presence of the skin were evaluated by applying the same surface continuous light illumination of $3.14 \mathrm{~W} / \mathrm{cm}^{2}$ between $\mathrm{L} 1$ and L2 corresponding to a position dorsal-lateral to PD \#5 (D). A peak light irradiance of $327.48 \mu \mathrm{W} / \mathrm{cm}^{2}$ was recorded at PD \#5 in the absence of the skin, and a peak light radiance of $5.68 \mu \mathrm{W} / \mathrm{cm}^{2}$ was recorded at both PD \#4 and PD \#5 in the presence of the skin. The difference of the light irradiances at PD \#5 at the absence and presence of the skin indicated a skin-alone light transmission of $\sim 1.7 \%$ at $980 \mathrm{~nm}$.

\section{Conclusions and Future Works}

We have developed a flexible nine-channel photodetection probe for deployment within the spinal canal of a dog after hemilaminectomy to measure transcutaneously transmitted PBMT irradiance at nine sites over an $\sim 8$-cm length of the spinal canal. The probe contained nine sets of PD conditioned individually to provide a response of $1 \mathrm{~V}$ per $7.58 \mu \mathrm{W} / \mathrm{cm}^{2}$ continuous irradiance at $850 \mathrm{~nm}$. This flexible nine-channel probe rendered the first set of simultaneous, nine-site intraspinal measurements of transcutaneously transmitted 980-nm PBMT light irradiation in a pilot cadaver dog. The maxima of the light irradiances measured by the nine PDs under an off-contact continuous surface irradiance of $3.14 \mathrm{~W} / \mathrm{cm}^{2}$ over an $\mathrm{L} 1$ to $\mathrm{L} 2$ vertebral position were $5.68 \mu \mathrm{W} / \mathrm{cm}^{2}$ with the skin and $327.48 \mu \mathrm{W} / \mathrm{cm}^{2}$ without the skin, respectively. Works have been on-going with six more cadaver dogs to evaluate transcutaneous transmission of PBMT light at various surface applications and on subjects of various sizes that are expected to be more relevant to clinical protocols.

\section{Disclosures}

This work has been supported by a grant from LiteCure LLC to D. Piao. L. De Taboada has financial interest in LiteCure LLC. No other conflicts of interest exist for this study.

\section{References}

1. C. E. Tedford et al., "Quantitative analysis of transcranial and intraparenchymal light penetration in human cadaver brain tissue," Lasers Surg. Med. 47(4), 312-322 (2015).

2. J. T. Hashmi et al., "Effect of pulsing in low-level light therapy," Lasers Surg. Med. 42(6), 450-466 (2010).

3. J. A. Zivin et al., "Effectiveness and safety of transcranial laser therapy for acute ischemic stroke," Stroke 40(4), 1359-1364 (2009).

4. M. Iusim et al., "Evaluation of the degree of effectiveness of biobeam low level narrow band light on the treatment of skin ulcers and delayed postoperative wound healing," Orthopedics 15(9), 1023-1026 (1992).

5. X. Wu et al., "Comparison of the effects of pulsed and continuous wave light on axonal regeneration in a rat model of spinal cord injury," Lasers Med. Sci. 27(2), 525-528 (2012).

6. C. C. Medalha et al., "Low-level laser therapy improves repair following complete resection of the sciatic nerve in rats," Lasers Med. Sci. 27(3), 629-635 (2012).

7. Y. Y. Huang et al., "Low-level laser therapy $(810 \mathrm{~nm})$ protects primary cortical neurons against excitotoxicity in vitro," J. Biophotonics 7(8), 656-664 (2014).

8. R. L. Buchaim et al., "Effect of low-level laser therapy (LLLT) on peripheral nerve regeneration using fibrin glue derived from snake venom," Injury 46(4), 655-660 (2015).

9. C. C. Cotomacio et al., "Dosimetric study of photobiomodulation therapy in 5-FU-induced oral mucositis in hamsters," J. Biomed. Opt. 23(1), 018003 (2017).

10. M. A. Hadis et al., "The dark art of light measurement: accurate radiometry for low-level light therapy," Lasers Med. Sci. 31(4), 789-809 (2016).

11. E. K. Ziago et al., "Analysis of the variation in low-level laser energy density on the crushed sciatic nerves of rats: a morphological, quantitative, and morphometric study," Lasers Med. Sci. 32(2), 369-378 (2017).

12. D. Piao, L. A. Sypniewski, and K. E. Bartels, "Challenges of transcutaneous laser application for the potential of photobiomodulation of the spinal cord at the scale of a large companion animal," Proc. SPIE 10048, 100480M (2017).

13. F. Tian et al., "Transcranial laser stimulation improves human cerebral oxygenation," Lasers Surg. Med. 48(4), 343-349 (2016). 